\title{
Participatory Collaboration in the Chinese Publishing Industry: Building Hierarchies to Express Value in a Virtual Collaborative Space
}

\author{
Ruoxing $\mathrm{Cao}^{\mathrm{a}}$, and Yufeng Zhang ${ }^{\mathrm{b}}$ \\ Department of Social \& Historical Sciences, University College London, London WC1E 6BT, \\ United Kingdom \\ a Ucsarc2@ucl.ac.uk, b Ucsayz2@ucl.ac.uk
}

\begin{abstract}
With the development of Internet technologies and the massive popularity of COVID-19, employee engagement in collaboration through digital technologies is increasing rapidly, by focusing on the basic practices of virtual collaborative work in the publishing industry, this essay (1) identifies new problems faced by traditional hierarchies in virtual collaboration from the perspective of participatory collaboration and critically assesses these problems. (2) attempts to propose a methodological solution that supports the stimulation of more participant creativity to facilitate collaborative creative activity in book products. The focus is on how to build more efficient work hierarchies in virtual spaces, making them a replicable means of gaining the ability to apply them in real work and organizations.
\end{abstract}

Keywords: Employee Engagement; Virtual Collaborative Space; Publishing Industry; Hierarchies; Problems; Propose Methodological Solutions.

\section{Introduction}

With the development of Internet technologies, technologies have emerged to support employee engagement in collaboration through digital technologies. The massive popularity of COVID-19 has also prompted companies to build online virtual spaces with digital software across time, space, and geography to encourage employee engagement in online collaboration. While effective in engaging employees in their work, this particular virtual collaboration space is still affected by the general problems of online technology. Specifically, in the publishing industry, for example, there is a risk of disorganization of the hierarchy that can be faced when working in a project-based orientation. As a result, perfect systems are pre-designed in the traditional way to solve possible work problems. Although pre-designed hierarchies greatly reduce the importance of this problem in the actual virtual space, the problem is still prevalent in everyday work, especially in start-ups. By focusing on the basic practices of virtual collaborative work in the publishing industry, this essay identifies new problems faced by traditional hierarchies in virtual collaboration from a participatory collaboration perspective, critically assesses these problems, and attempts to propose methodological solutions.

\section{Participatory Collaboration for Virtual Space and Practical Applying in Publishing Industry}

\subsection{Participatory Collaboration for Virtual Spaces}

In the context of the massive emergency crisis of COVID-19, work-oriented virtual collaboration environments are becoming widely used by businesses. Virtual spaces draw on computer technology's 'abilities to bridge time and to bridge space' (Baecker et al., 1995), 'can enhance the sametime/different-place interaction of existing tele- and video-conferencing and instant messaging tools by providing synchronous virtual meeting support within a space that acts as a persistent repository of informational objects representing resources used and generated by the collaboration, and moreover in such a way as to complement the rich potential of web technologies as a means for asynchronous collaboration' (Tate 2014, 2). In its origins, the Virtual Collaboration Space (VCE) was used to support remote participants to collaborate in response to unexpected crisis events such as the 
2004 Indian Ocean tsunami. Today, virtual networking technologies are becoming the technological backbone for corporate employees to practice their expertise as large numbers of users enter virtual collaboration spaces to complete their daily work activities. Not only that, but the everyday workspace operating in a technological context has also had a reverse effect on the hierarchy, with employees 'use technical artifacts to perform their tasks (even natural language is such an artifact, properly speaking), and, moreover, apply particular, perhaps socially or culturally mediated, physical and mental techniques in their use of these artifacts' (Tate 2014, 4).

\subsection{Practical Applying in Publishing Industry}

The book publishing industry, as an industry where commerce and art are intertwined, has a more socially and culturally mediated quality to its project-oriented work. The publishing team, a combination of editors, writers, illustrators, and publishing managers, needs to focus not only on getting the job done but also on communicating on a culturally cognitive level. With the mass adoption of virtual collaboration technology, the publishing industry has been able to bring together artists and writers from around the world to work on the same publishing project, across time and space. The author's publishing start-up, for example, is oriented towards book projects, setting up micro-teams for contracted artists and writers and editors to develop books through participatory collaboration in Google Docs. The company provides hardware and software support for artists and authors and organizes regular online company meetings through Tencent Meetings. In practice, the collaboration focuses on the different artists communicating with each other, collectively, to establish a default hierarchy within the virtual space. This process of activity is not bound by company systems but is influenced by project characteristics and the cultural preferences of the staff. As a result, the influence of traditional publishing company hierarchies is diminished in the practice of collaboration in virtual spaces.

\section{Problems and Solutions of Virtual Participatory Collaboration}

\subsection{Analysis of Current Status}

As with the process of building all enterprise hierarchy models, in order to ensure the orderly operation of collaboration in the virtual space, companies tend to base their work on the traditional hierarchy building model, develop work norms for employees working in the virtual space and delineate the hierarchy in the virtual space. However, 'In dynamic social situations, applying the conventional deductive analytic framework is likely to produce invalid results. A better approach is to radically switch paradigms and rely on the systemic paradigm, working inductively and grounding our emergent understanding of work practices in iterative recourse to the data' (Brigitte 1996, 21). Without timely adjustments to traditional hierarchical frameworks, new problems may arise for collaboration in virtual spaces.

The new issues mentioned here are particularly salient in the context of virtual collaboration in the publishing industry, i.e., publishing projects carried out in virtual spaces are influenced by the cultural preferences of the project features and artists, thus creating an impact on the traditional hierarchy. Firstly, unlike ordinary corporate employees, artists and writers work with a creative orientation and a strong sense of self. Working in a virtual space may amplify this sense of individuality, while 'these medium lacks reinforcing cues such as eye-contact and properly synchronized head-nods (along with other forms of accurate gestural communication) to confirm a speaker has the audience's attention or even that the members of the audience are actually 'in attendance' at their computers' (Tate 2014, 8). Thus, the lack of reinforcement cues and the increased sense of individuality make communication between editors and writers massively more costly.

In addition, the participatory collaboration that takes place in the virtual space weakens the employees' sense of belonging to the company, James defined social dependency in his South African study, stating that 'dependence was not simply bondage or unfreedom (...) It was actually the existence of possibilities for hierarchical affiliation that created the most important forms of free 
choice (...) this was a way not only of structuring society, but also of constructing persons' (Ferguson 2013, 226). People, as nodes in a system of relationships, construct society through interdependence and mutual respect. Hierarchies and social hierarchies are formed through the construction of hierarchical dependencies, 'it was the principal mechanism for achieving social personhood' (Ferguson 2013, 226). Company hierarchies are similarly constructed by building hierarchical dependency and group belonging among employees to maximize the effective functioning of the system. In participatory collaboration in virtual spaces, particularly in publishing companies with strong links to artists, it is crucial to shaping hierarchical structures with a sense of belonging and dependency. While a great deal of institutional design has attempted to mitigate this problem, collaboration in virtual spaces again magnifies the problem, and reliance on traditional hierarchies alone does not fully address it.

\subsection{Proposed Solutions in the Future}

\subsubsection{Building Virtual Utopian Workspaces for Employees}

The traditional hierarchy does not meet the needs of working in a virtual space, but there is no single solution that perfectly solves all the problems faced by companies in a virtual space. In fact, there are multiple attempts to solve the problem of collaboration in the virtual space by focusing on the publishing industry alone. However, if the focus of the problem is on the literary qualities of the publishing industry, the construction of virtual utopian spaces opens more possibilities for artists to communicate with the business community. In the virtual space, publishing editors combine socially realistic practices to 'emphasis on what is doable and viable given the conditions of the present' (Cooper 2013, 4), while encouraging artists to 'capture a sense of hope and potential, in that they anticipate something more, something beyond and other to what they can currently realize' (Cooper $2013,4)$. In this sense, the virtual utopia creates a better working space for artists in which they can critique the current world and at the same time integrate their ideas with social reality with the help of the company. Not only that, 'everyday utopias don't simply enact new practices, responding to participants' prior interests and sense of how things should be. Everyday utopias also bring about (or seek to bring about) new forms of normalization, desire and subjectivity' (Cooper 2013, 5). The Virtual Utopia space not only responds to the creative ideas and feelings of the employees but also draws on the company's resources to create a creative atmosphere for the virtual space and to inspire the artists' work.

\subsubsection{Systematic Mobility}

As a publishing company, in a project-oriented virtual utopian space, the power of employees and artists is in a state of flux, and the hierarchy in this space is fluid. Therefore, companies need to identify the core members of the book development process in the virtual workspace and establish the status of intermediaries for them, responsible for structuring the internal hierarchy of team members and communicating with the external world. Cooper defined such 'intermediaries' as 'mediators' of the utopian world, 'the most explicit mediations are performed by creators and (fictional) visitors - in other words, by those who bridge the world of utopia and the world outside' (Cooper 2013, 39). Traditional publishing companies tend to regulate the publishing process by making the intermediary explicitly a publishing manager or editor. In the virtual space, the author's publishing start-up attempted to design a fluid intermediary identity in which the book selection was the core, and the employee who proposed the selection acted as the intermediary connecting the workspace to the outside world and was responsible for constructing the hierarchy of that virtual workspace. In this design, publishing editors, writers, painters, and other company members can be at the heart of the project, with the leadership team merging imagination and practice through participatory collaboration. 


\section{Potential Challenges of Utopian Collaborative Space}

Participatory collaboration in virtual spaces can be a practical solution to the problem of solidifying hierarchical structures in traditional work models and making them fluid. The shift to virtual utopian spaces for publishing work serves as a possible candidate solution for building a sense of dependency and belonging for employees, while proposing an optimized solution to hierarchical structures. However, this paper focuses its analysis on the publishing industry, which is highly artistic, and in an analysis using the author's company as an example, it shows the practical problems that publishing start-ups encounter in collaborating in virtual spaces and presents an attempted idea for building utopian spaces. However, the practical challenges that companies will face are how to build virtual utopian spaces in their work and how to keep the flow of hierarchical structures in line with the stability of their operations.

In addition, it is worth noting that virtual spaces built around the corporate context are public workspaces for employees, but virtual space collaboration makes it impossible for companies to monitor employees' private spaces and thus the actual level of employee involvement in the work process. Once the hierarchical structure of mobility within the utopian space tends to become unstable, companies are likely to lose credibility, which leads to a decrease in employee engagement at work. 'People not only arranged parallel events at ideological functions, but also tried to arrange as much free time for themselves as possible within the official sphere' (Yurchak 1997, 184). The instability of hierarchical structures can cause companies to lose the basis of their existence, and the participatory collaboration of virtual spaces makes it difficult for modern companies to circumvent this potential problem, while the construction of everyday utopian spaces is likely to increase the risk of this problem arising.

\section{Summary}

Against the backdrop of the global pandemic of COVID-19, different companies in the publishing industry are building their own virtual space work systems through a practice approach. Using the author's own start-up as an example, this essay provides a means for publishing companies to attempt to address the potential risks and challenges of working online in publishing companies by designing a virtual utopian space. However, it cannot be denied that there are still practical operational problems with the tool. In order to support the continued operation of the utopian space in participatory and collaborative publishing work practices, the author will continue to experiment with running virtual utopian spaces, identifying and documenting practical problems encountered in his work, and using anthropological methods to try to transform them. It is worth believing that this work has the potential to help publishing companies create more efficient hierarchies and maximize the creativity of their employees.

\section{References}

[1] Jordan, Brigitte. 1996. "Ethnographic Workplace Studies and CSCW." In: The Design of Computer Supported Cooperative Work and Groupware Systems, edited by Dan Shapiro, Michael Tauber, Roland Traunmüller, 17-42.

[2] Baecker, R.M., Grudin, J., Buxton, W.A.S., Greenberg, S. 1995. Groupware and Computer-Supported Cooperative Work. In R.M. Baecker, J. Grudin, W.A.S Buxton, S. Greenberg (eds.) Readings in HumanComputer Interaction: Toward the Year 2000, Morgan Kaufmann.

[3] Tate, Austin. 2014. "Virtual Collaboration Spaces: Bringing Presence to Distributed Collaboration." Journal Of Virtual Worlds Research 7, Issue 2: 1-14. https://jvwr-ojs-utexasstage. tdl. org/ jvwr/ index. $\mathrm{php} / \mathrm{jvwr} / \mathrm{article} / \mathrm{view} / 7090$.

[4] Ferguson, Peter. 2013. "Declarations of Dependence: Labour, Personhood, and Welfare in Southern Africa." Journal of the Royal Anthropological Institute 19: 223-242. https://www-jstor-org. libproxy. ucl. ac.uk/stable/42001576? sid=primo. 
[5] Cooper, Davina. 2013. Everyday Utopias: The Conceptual Life of Promising Spaces. Duke University Press.

[6] Yurchak, Alexei. 1997. "The Cynical Reason of Late Socialism: Power, Pretense, and the Anekdot." Journal of Public Culture 9: 161-188. https://read-dukeupress-edu. libproxy. ucl. ac.uk/ public-culture/ article/ 9/2/161/32247/The-Cynical-Reason-of-Late-Socialism-Power. 